\title{
La extensión artística-cultural y deportiva en la formación integral de los estudiantes universitarios
}

(C) Copyright 2021. Universidad Nacional Autónoma de Nicaragua, Managua (UNAN-Managua)

Todos los derechos reservados

\section{The artistic-cultural and sports extension in the comprehensive training of university students}

Wilmer Martin Guevara

FAREM-Carazo

wguevara@unan.edu.ni

https://orcid.org/0000-0002-5657-8114

\section{Resumen}

El presente artículo destaca la importancia de las actividades culturales y deportivas de la Facultad Regional Multidisciplinaria de Carazo (FAREM-Carazo) en el marco de la extensión universitaria como una de las funciones sustantivas de nuestra universidad. Los objetivos que guiaron este proceso fueron: 1) Diagnosticar el estado actual de las disciplinas artísticas y deportivas de la FAREM-Carazo y 2) Reflexionar sobre el aporte de estas actividades en la formación integral de los estudiantes de la FAREM-Carazo. La revisión documental y las entrevistas a los artistas, atletas y entrenadores han sido el método y técnica propicia para compilar la información; por su parte, la triangulación de datos por informantes ayudó a validar los aportes de los entrevistados.

Los resultados reflejan una participación activa del estudiantado, a pesar de que no se cuenta con las instalaciones ni recursos óptimos para la práctica de las disciplinas artísticas y deportivas. Además, los estudiantes reconocen que pertenecer a una disciplina artística o deportiva les otorga cierto estatus de reconocimiento en su comunidad, la facultad y en su grupo particular, además de aportar en ellos (as) la capacidad de trabajar en equipo, seguridad escénica y liderazgo. Se puede concluir que las actividades culturales y deportivas juegan un papel preponderante en el desarrollo integral del estudiantado y de la sociedad en su conjunto, en especial de los sectores menos favorecidos, preservando, difundiendo y poniendo a su alcance los servicios y bienes culturales y recreativos que nacen de la misma sociedad.

\section{Palabras claves}

Actividades culturales y deportivas, equipamiento e infraestructura cultural y deportiva, extensión universitaria, formación integral, promotor cultural y deportivo, vinculación.

\section{Summary}

This article highlights the importance of the cultural and sports activities of the Carazo Regional Multidisciplinary 
Faculty (FAREM-Carazo) in the framework of university extension as one of the substantive functions of our university. The objectives that guided this process were: 1) To diagnose the current state of the artistic and sports disciplines of the FAREM-Carazo and 2) To reflect on the contribution of these activities in the integral formation of the students of the FAREMCarazo. The documentary review and the interview with the artists, athletes and coaches have been the favorable method and technique to compile the information, meanwhile the triangulation of data by informants helped to validate the contributions of the interviewees.

The results reflect an active participation of the student body, despite the fact that there are not optimal facilities or resources for the practice of artistic and sports disciplines. In addition, students recognize that belonging to an artistic or sports discipline gives them a certain status of recognition in their community, the faculty and in their particular group, in addition to providing them with the ability to work as a team, stage safety and leadership. It can be concluded that cultural and sports activities play a preponderant role in the integral development of the student body and of society as a whole, especially of the less favored sectors, preserving, disseminating and making cultural and recreational goods and services available to them. that are born from the same society.

\section{Keywords}

Cultural and sports activities, cultural and sports equipment and infrastructure, university extension, comprehensive training, cultural and sports promoter, bonding.

\section{Introducción}

Este trabajo demuestra la contribución de las actividades culturales y deportivas en la formación integral de los estudiantes universitarios, en cuanto a su salud física, mental y emocional, así como en su proyección comunitaria y laboral. También, determina el impacto positivo de las actividades culturales y deportivas como formas de hacer extensión que tienen un impacto hacia adentro y hacia afuera de la universidad, además, refleja cómo -a pesar de su importancia- las autoridades correspondientes, abonan poco para el desarrollo satisfactorio de estas disciplinas.
Para cumplir con los objetivos planteados anteriormente, se estudió en el caso de la Facultad Regional Multidisciplinaria de Carazo; entrevistando a los protagonistas (atletas, artistas, entrenadores, promotores culturales) y revisando los registros de actividad cultural y deportiva que publica el boletín informativo Torreón Universitario de la FAREMCarazo, durante el período 2018-2020. Se desarrollo en primer lugar, una breve ubicación de las actividades de extensión cultural en el ámbito de estudio, luego una descripción sobre el estado actual de las instalaciones deportivas y culturales, y la situación en cuanto a los recursos que tienen para desarrollar sus actividades recreativas; además, se hizo un contraste con las opiniones que los protagonistas (artistas y deportistas) expresan sobre este panorama. Finalmente, para ubicar en este artículo, el desarrollo de las actividades culturales y artísticas como forma de hacer extensión, se realizó una breve reseña de esta perspectiva.

La inclusión de la extensión como parte de la vida de las instituciones académicas se logró con la reforma de Córdoba en 1918, y solo ha sido asumida en Latinoamérica de forma paulatina. Esto explica por qué a lo largo de su historia "la extensión ha sido la función menos atendida en las instituciones a nivel superior" (Ángeles, 1992 citado por Fresan Orozco, 2004, p. 48). Otro de los problemas de la extensión universitaria es la falta de inteligibilidad tanto para la comunidad externa como para los propios universitarios, es decir, que existe poca valoración o identidad con esta función y lo que representa en términos de formación del estudiantado y de vinculación con la sociedad (Ortega y Villa 2003 citados por Fresan, 2004).

En Nicaragua, desde los tiempos del neoliberalismo, Tünnermann (2002) reconocía que la extensión universitaria es la función a la que menos se ha prestado atención e identificaba como elementos de esta problemática, la relevancia de la docencia frente a la extensión y la poca relación entre ambas, situación generada por el valor secundario que se le otorga a la segunda, así como el poco financiamiento de recursos asignado a estas tareas, la insuficiencia de personal dedicado a su funcionamiento y por último, el "paternalismo cultural" de la difusión asumido especialmente por las autoridades superiores.

En el ámbito de la operación de la extensión universitaria y, particularmente en el de la difusión de la cultura, ocurren también grandes contradicciones. Por una parte, quienes tienen a su cargo la organización 
de las actividades artísticas se mantienen alejados del ámbito educativo y de la investigación. Por otro lado, los profesores universitarios recurren poco al apoyo de los promotores culturales para la organización de actividades artísticas, eventos académicos o ciclos integrales, porque difícilmente encuentran una respuesta a sus necesidades. Esta incomunicación, dispersa los esfuerzos y los recursos que se invierten en su función y conspira contra la posibilidad de lograr la formación integral de los alumnos que implica considerar, además de los contenidos curriculares, la atención a un conjunto de actividades relacionadas con el desarrollo físico, ideológico, cultural y emocional del estudiante. (Fresan Orozco, 2004, p. 51)

Durante los gobiernos neoliberales en Nicaragua, la extensión tenía un carácter "culturalista" e intrauniversitario y no lograba una comunicación de doble vía con la sociedad, porque se reducía a la presentación de grupos artísticos a lo interno de los recintos universitarios (Tünnermann, 2002). A este desconcierto conceptual derivado de la falta de identidad de la extensión universitaria se suma la resistencia de precisar los objetivos de esta función. Por ello, distintos sectores universitarios cuestionan los recursos invertidos en las actividades culturales y en la extensión universitaria (Fresan Orozco, 2004, p. 52).

A nivel nacional, la Ley 89 de Autonomía Universitaria, es una regla básica que menciona la noción de Extensión Universitaria en los fines y objetivos el artículo 6) punto 6) establece, entre otras cosas: organizar la Proyección Social, la Difusión Cultural y la Extensión. El espíritu de esta ley se recoge ampliamente en la Política de Extensión y Proyección Social Universitaria de la UNAN-Managua (2018), en la cual dedica dos áreas completas; 1) área de Arte y Cultura y 2) Área de Educación Física y Deporte, en los que reconoce el valor de la cultura y el deporte como áreas de formación integral.

Sin embargo, se abordará el tema a partir del informe final del proceso de Autoevaluación Institucional con fines de mejora, realizado en 2013, que reconoce entre otras cosas, la falta de infraestructura para la práctica de deporte y cultura como una de las limitantes de la UNAN-Managua, lo cual se agrava en las facultades regionales como la FAREM-Carazo.

\section{Antecedentes históricos de la Facultad Regional Multidisciplinaria de Carazo}

La Facultad Regional Multidisciplinaria de Carazo, tiene sus antecedentes en la Escuela de Ciencias de la Educación -única en su género en Nicaragua-, a propuesta por el entonces rector de la UNAN, Dr. Mariano Fiallos Gill, quien identificó tal necesidad, porque un grupo de intelectuales Jinotepinos habían iniciado de forma empírica esta actividad con el asesoramiento y apoyo de expertos en temas de la educación, del club rotario de la UNESCO en la ciudad (FAREM-CARAZO, 2015). El Dr. César Amalla, médico de la ciudad y director del Hospital Regional Santiago de Carazo, fue el primer director del Centro de Estudios, que inicio con la carrera de Psicopedagogía, pero debido a sus ocupaciones médicas, Amalla pronto sería sucedido por Juan Sánchez Flores. Una característica de estos directores y su reducido claustro docente, es que en los primeros años fueron Ad Honorem.

En otras palabras, la universidad en Carazo, desde su nacimiento se gestaba como una actividad de extensión académica-social en sí misma, al ver la alta demanda de jóvenes que no podían acceder a los centros prestigiosos del departamento, como la Escuela de Señoritas ubicada en la ciudad de San Marcos y la Normal Franklin D. Roosevelt en Jinotepe. El 11 de noviembre de 1960 -cuando la Escuela de Ciencias de la Educación aún no tenía un lugar permanentesucede la toma de los cuarteles de Jinotepe y Diriamba como parte de las acciones del Movimiento Juventud Patriótica Nicaragüense, uno de los grupos opositores que buscaba derrocar la dictadura militar somocista, instaurada en Nicaragua desde 1939.

En 1981, deja de funcionar la Escuela de Ciencias de la Educación que era una extensión de la UNAN-Managua, pero el 11 de noviembre de 1990, exactamente 30 años después de la toma del cuartel en Jinotepe, se inaugura en el antiguo Torreón de la Guardia Nacional, el Centro Universitario Regional de Carazo, por gestiones de un grupo de personas, encabezadas por el Dr. Armando Rodríguez (FAREM-CARAZO, 2015). Los primeros problemas que enfrentaron eran de tipo económico, pues no se contaba con un presupuesto establecido para el recién formado recinto universitario. 


\section{Surgimiento de las actividades deportivas $y$ culturales en la FAREM-CARAZO}

Desde su nacimiento en los años 90, como Centro Universitario Regional de Carazo hasta el 2006 la universidad centraba su actividad de extensión -aunque de forma limitada- en lo académico. El Voleibol y el Basquetbol figuran entre los primeros deportes practicados de manera formal. En palabras de Marlon Narváez (2020), la disciplina de Voleibol se practica desde 1994, e inició con un grupo de jóvenes entre los que destacaba el maestro Raúl Medrano Chávez, Dallan, Mario Chan y Alejandro, conocido como "La Chana". Entre 1998 y 2001, toma los entrenamientos el profesor Pablo Aburto. Luego, la profesora Ana Laura García, se hace cargo del equipo de Voleibol masculino y femenino. Otra disciplina deportiva se suma en 2003, el Tae Kwon Do, cuyo primer instructor fue Jimmy Horacio Zúniga Baltodano (actualmente instructor del IND en Diriamba).

\section{Después de 2006}

El 2006 significó un parte aguas en las actividades de extensión cultural universitaria. Con la administración del Dr. Pedro Aburto Jarquín, director del entonces CUR-Carazo, quien además era músico, se formalizan dos disciplinas artísticas: la música y la danza, que antes solo se promovía de manera esporádica en las actividades conmemorativa de la universidad. Henry Narváez y Marlon Matus Aronson, dos reconocidos músicos jinotepinos, son llamados por el doctor Aburto e inician a impulsar la música coral y el canto de trova.

Por su parte, Wilmer M. Guevara, entonces estudiante de la carrera Turismo Sostenible, ya desde las aulas de clase organizaba a los estudiantes de su carrera y a los alumnos de economía agrícola para promover las danzas nacionales en las actividades conmemorativas de la universidad, es así delegado para asumir el grupo de danza que inicialmente se llamó "Flor de Sacuanjoche". Los estudiantes de otras carreras se sumaron al proyecto y muchos de ellos desde la educación secundaria se integraron antes de entrar a la universidad.

En 2009 se suman otras disciplinas deportivas de tipo salón: El Ajedrez y el Tenis de Mesa, a cargo del maestro Sergio Iván Carranza, ajedrecista con una increíble trayectoria, difícil de superar en el ámbito nacional y centroamericano. Estas disciplinas proyectan de inmediato a la universidad y además integran a la niñez de colegios de primaria del departamento de Carazo, como una forma de hacer extensión. En el caso del Ajedrez, la FAREM-Carazo se mantiene dentro de la élite nacional de este deporte, cuidando de forma muy celosa la renovación de los ciclos de atletas que suceden entre los 4 y 6 años.

En este mismo año, se suma la disciplina de Karate con el instructor Martín Largaespada, con quien han ganado juegos internacionales como los de la UNACH en Honduras, en donde se obtuvo en 2016, la medalla de oro de ese torneo. Paradójicamente, el voleibol, uno de los deportes que hasta 2006 se había impulsado grandemente, queda sin entrenador. Sin embargo, un cambio importante en 2008 para la disciplina de Tae Kwon Do, fue la contratación de Mario Zabala -actual entrenador de la selección nacional- que ayudó a la proyección del equipo.

En el caso de la danza como disciplina artística, inicia un proceso de metamorfosis. En 2007 incursiona en los ritmos latinos, por lo cual se llegó a tener dos agrupaciones artísticas de danza; Flor de Sacuanjoche y Corazón Latino, sin embargo, en 2008 las agrupaciones se fusionan en una sola, llamada Corazón Latino y se perfilan hacia los ritmos urbanos, estilo que los definió con sello propio y que incidió para que las demás facultades de la UNAN-Managua también incursionaran en este género, al punto que en 2017 para el FICCUA, el montaje presentado en danza variedad, fue en ritmos urbanos y el coreógrafo fue el maestro Wilmer Guevara, entonces promotor cultural de la FAREM-Carazo.

\section{1: Otros cambios se avizoran}

Nuevos cambios venían para la extensión cultural y el deporte en la FAREM-Carazo. En 2011, con el Dr. Gerardo Raúl Arévalo Cuadra como decano, Francisco Rodríguez asume la disciplina de Tae Kwon Do, catapultando el deporte a sus mejores tiempos, uno de los momentos inolvidables fue la participación de los atletas en los juegos del ALBA en Venezuela, en el que se obtuvo medalla de Plata. También es importante destacar la cantidad de atletas que aporta esta disciplina a la selección nacional, desde esa fecha.

Solo dos años después, en 2013 contratan a Marlon Narváez para atender la disciplina de Voleibol femenino y masculino, y son entre los deportes, uno de los que genera gran movilidad de estudiantes en el ámbito nacional, llevando la presencia de la facultad, hasta 
las costas de los dos grandes mares de Nicaragua a través del voleibol de playa. En 2013, también se genera otro cambio importante, Emilio Reyes -músico del conservatorio nacional- asume el área de música, e incursiona en diferentes géneros musicales, entre ellos religiosos y populares, con un acento en la cumbia, la salsa, la música nacional y de trova.

\section{El estado actual}

En el informe final del proceso de Autoevaluación Institucional con fines de mejora, realizado en 2013, se reconoce que una de las limitantes de la UNANManagua es la falta de infraestructura para la práctica de deporte y cultura, y la FAREM-CARAZO no es la excepción. Se cuenta con un polideportivo que se ubica en el recinto Augusto C. Sandino, que está fuera del centro de la ciudad, lo cual limita la asistencia de los atletas, además de no prestar las condiciones óptimas para los entrenamientos. A lo interno de la facultad, en ocasiones, se presta el auditorio para los entrenamientos, aunque en los últimos años se ha prohibido su uso, por razones de mantenimiento de las instalaciones físicas.

Por otra parte, se ha designado el segundo piso del edificio Rafael Sánchez Richardson -donde funciona la biblioteca-, para las prácticas de Tae Kwon Do y danza, pero es un espacio reducido, con poca ventilación e iluminación, que no permite un entrenamiento satisfactorio, además de estar en proceso de demolición por antigüedad. En palabras de Verónica Ríos (2020) -integrante del equipo de Tae Kwon Do- "El área donde practicamos no es lo idóneo; el ambiente, los recursos y los materiales no son los adecuados, y los pocos con los que contamos no son propios de la institución", Mario Urtecho (2020), también de Tae Kwon Do detalla "no tenemos tatami, yogui o pechaque".

Algunos docentes coinciden con los estudiantes respecto a la falta de espacios, que limita la participación de los jovenes; sin embargo, el maestro de ajedrez Iván Carranza (2020) refiere que, como forma de mitigación han optado por realizar los entrenamientos en lugares externos a la facultad. Otras disciplinas como el Voleibol o el Karate hacen lo mismo, prestando espacios públicos como el polideportivo municipal y la antigua estación de ferrocarril. En cuanto a los recursos, la situación no cambia mucho. Son pocos y se carece de presupuesto anual. Se suma a esto, el saqueo que sufrió la universidad en 2018 por grupos armados que participaron en el intento fallido de golpe de Estado en Nicaragua. Así mismo, la participación de los estudiantes se ha visto mermada desde 2018 por el acontecimiento citado, y desde marzo de 2020, por el efecto de la pandemia COVID-19, a pesar de que nunca se suspendieron las actividades normales en la universidad.

Algunas estrategias de mitigación frente a la carencia de espacios físicos y recursos, utilizadas por los instructores y sus alumnos es la adquisición de materiales por su propia cuenta, aunque esto resulte poco satisfactorio para los estudiantes -que son de escasos recursos-y además, limita el crecimiento de los grupos deportivos o culturales. En el caso de Voleibol, nos dice su entrenador, que realiza como acción de mitigación, alianzas con el Instituto Nicaragüense de Deporte (IND), el movimiento Alexis Argüello, la Juventud Sandinista, la Alcaldía del municipio y grupos de amigos del Voleibol, que en general les apoyan con la gestión de equipamiento, lo cual es del conocimiento de la vice decanatura (responsables de extensión en las facultades, según los estatutos de la UNAN-Managua). Otra dificultad a la que se enfrentan los estudiantes, deportistas y artistas,es a la cultura docente, que poco comprende la importancia de estas actividades para la formación profesional, y que en algunos casos las consideran como actividades de ocio, que no son provechosas.

A pesar de las realidades complejas en la que se desarrolla la cultura y el deporte como actividades de extensión, la participación es altamente dinámica, tal como se demuestra en la tabla 1, en la cual, se presenta el registro de las actividades culturales y deportivas entre enero de 2018 hasta octubre de 2020; sin embargo, se deben tomar en cuenta las siguientes consideraciones:

- Se refleja solo las actividades reportadas y publicadas en el boletín oficial de la facultad "Torreón Universitario" y no las que reporta trimestralmente cada uno de los promotores culturales $\mathrm{o}$ entrenadores de deporte a la facultad. Eso se debe a que el boletín, es el medio más oportuno para citar de forma objetiva las evidencias.

- Se incluyen las actividades deportivas sindicales y la gimnasia laboral. 
Las actividades se clasificaron en:

- Públicas: las actividades vinculadas a instituciones del Estado, organizaciones territoriales u otras universidades del CNU.

- Privadas: las actividades vinculadas al sector privado.

- Internas: las actividades realizadas en el seno de la UNAN-Managua y cualquiera de sus facultades; en la capital o las regiones.

- Externas: las actividades realizadas fuera de las instalaciones de la UNAN-Managua y sus recintos (central o regionales).

- Nacionales: las actividades realizadas en Nicaragua.

- Internacionales: las actividades realizadas fuera del país o en Nicaragua siempre y cuando, tengan vinculación con entidades extranjeras.
Si analizamos verticalmente la tabla 1, sobre el registro de las actividades culturales y deportivas de la FAREMCarazo, de acuerdo a la clasificación de las actividades se puede anotar lo siguiente:

- De las actividades registradas en el periodo 20182020, 37 de las 43 se realizaron en coordinación con el sector público.

- De las 43 actividades, 32 se realizaron a nivel interno, es decir, entre las facultades de la UNANManagua.

- Solamente se registró una actividad de carácter internacional.

De lo anterior podemos inferir que la participación de la universidad se realiza más en el sector público y se mantiene ensimismada, es decir, falta mayor vinculación con el exterior de la institución, pero además falta trabajar más la internacionalización del deporte y la cultura.

\begin{tabular}{|c|c|c|c|c|c|c|c|} 
Año & Actividad & Pública & Privada & Interna & Externa & Nacional & Internacional \\
\hline \multirow{4}{*}{2018} & Culturales & 2 & - & 1 & 1 & 2 & - \\
\cline { 2 - 8 } & Deportivas & 6 & 1 & 2 & 5 & 6 & 1 \\
\cline { 2 - 8 } & & 8 & 1 & 3 & 6 & 8 & 1 \\
\hline \multirow{3}{*}{2019} & Culturales & 3 & - & 3 & - & 3 & - \\
\cline { 2 - 9 } & Deportivas & 11 & 2 & 11 & 2 & 13 & - \\
\cline { 2 - 9 } & & 14 & 2 & 14 & 2 & 16 & - \\
\hline \multirow{3}{*}{2020} & Culturales & 1 & - & 1 & - & 1 & - \\
\cline { 2 - 9 } & Deportivas & 14 & 3 & 14 & 3 & 17 & - \\
\cline { 2 - 8 } & & 15 & 3 & 15 & 3 & 18 & \\
\hline
\end{tabular}

Tabla 1. Registro de las actividades culturales y deportivas de la FAREM-Carazo, publicadas en el boletín Torreón Universitario de la FAREM-CARAZO, entre 2018 y 2020. 
También se puede observar en la misma tabla, la diferencia sustancial entre la cantidad de actividades de deporte con relación a las actividades culturales. Esto se determina en parte, porque existen más disciplinas deportivas que culturales. Las disciplinas deportivas son: Voleibol, tenis de mesa, ajedrez, karate y tae kwon do, más otras actividades que se practican de forma esporádica como el basquetbol, atletismo, béisbol y fútbol. Por la parte de cultura, solo se cuenta con dos disciplinas: música y danza. Cada una de estas disciplinas, culturales o deportivas, por lo general mantienen inscritos entre 25 a 30 estudiantes, en dependencia de los horarios académicos por semestre. Por lo cual, la participación de los estudiantes en deporte y cultura se mantiene en un rango entre 250 a 300 estudiantes en cada semestre: un 16\% de la matrícula de la facultad.

Otro aspecto relevante a observar en la tabla 1, es que las actividades tienen su mínimo nivel de participación en 2018, debido a la desestabilización generada en el país por el intento de golpe de Estado y su ensañamiento con la universidad pública, por grupos armados que saquearon la institución, luego en 2019 hay una recuperación que sigue en ascenso en 2020 a pesar de la pandemia de COVID-19.

Finalmente, si tomamos como ejemplo, el año 2020 en la tabla 1, es necesario destacar que la suma de las actividades (dieciocho en este caso) refleja cerca del $20 \%$ de las actividades que realizan anualmente las disciplinas culturales y deportivas. Esta situación obedece a dos razones; 1) el boletín publica las actividades realizadas por las instancias organizativas, pero no la participación cultural o deportiva, 2) Las disciplinas que menos reportan actividades al boletín son las de cultura; en el registro de los boletines (20182020), no se registró ningún evento del grupo musical y solo uno del grupo de danza. En este punto, se debe mencionar que, según los promotores culturales, muchas de las actividades no son reportadas de forma oportuna.

\section{Incidencias de las actividades culturales y deportivas en la formación integral}

Desde la visión de los estudiantes, las actividades culturales y deportivas inciden en ellos, en dos direcciones; la primera, en la formación de valores como la disciplina, el trabajo en equipo y el liderazgo, pero además a no incursionar en "malos caminos" como las adicciones o la integración a grupos delincuenciales. De forma concreta, Madison (2020) - estudiante de primer año de medicina que practica karate- expresa que ayuda a "la salud mental, ya que como adolescentes tendemos a tener problemas de presión (por los trabajos o la familia) o ansiedad, y mantener nuestra mente ocupada puede ser de muchísima ayuda". Estos valores se reflejan según sus propias palabras en "la puntualidad y responsabilidad al momento de entregar un trabajo asignado y en la solidaridad entre los compañeros de clase", según afirma Verónica Ríos (2020) —estudiante de segundo año de Trabajo Social que practica Tae Kwon Docontinúa: "apoyándonos mutuamente, creciendo juntos".

En segundo lugar, el reconocimiento social que tienen los artistas y atletas frente a sus compañeros y el resto de la sociedad, es una plataforma para destacar socialmente, y que además les abre oportunidades laborales, ya sea en el ámbito de la disciplina cultural o deportiva que practiquen o en la especialidad de su carrera profesional. Lenar Cabeza (2020) -estudiante de primer año de economía que practica karateexpone "este deporte nos permite abrir puertas, ya sea dentro de la universidad o afuera, ¿Cómo abrir puertas? Darnos a conocer a través de ello, optar a un futuro cargo o un puesto".

Desde la perspectiva de los promotores culturales y deportivos, explica Francisco Rodríguez (2020) -entrenador de Tae Kwon Do- que los deportes contribuyen con la disciplina del estudiante, que se traducen en puntualidad, respeto y tolerancia, que los encamina al buen vivir como personas, además, añade Rodríguez: "cuando en 2017 aún se escogía a la excelencia deportiva y cultural, se le exigía al atleta promedios académicos de $90(. .$.$) y la FAREM-Carazo$ siempre obtenía varios reconocimientos de ese tipo, lo cual evidenciaba que los muchachos son excelentes estudiantes y excelentes atletas".

Por su parte, Emilio Reyes (músico) y Eduardo Delgado (futbol), dejan ver, como ya lo hicieran los estudiantes, que estas actividades de extensión, ayudan a los estudiantes a establecer vínculos para insertarse con mayor facilidad en el campo laboral de su disciplina, o como lo comenta David Martínez -un estudiante de computación que pertenece al grupo de cultural: "con la danza me di a conocer y me han buscado para dar clases de danza en colegios y mucha gente me conoce por esto". Así mismo, Emilio Reyes destaca que estas 
actividades (culturales y deportivas) mantienen a los estudiantes alejados de las drogas y otras prácticas nocivas para su salud, por lo cual infiere que este beneficio también es para la familia, los vecinos, la sociedad, que pueden estar más tranquilos, porque el muchacho mantiene la mente ocupada en actividades sanas y productivas.

\section{Conclusión}

Finalmente se puede decir, que las actividades culturales y deportivas como prácticas de extensión, tienen un impacto positivo en diferentes niveles: institucional, personal, familiar, comunitario, artístico de salud física, mental y emocional de la comunidad

\begin{tabular}{|c|c|c|c|c|c|c|c|}
\hline $\begin{array}{l}\text { Actividad } \\
\text { (Ajedrez) }\end{array}$ & $\begin{array}{c}\text { Fecha I } \\
\text { Sem. } 2020\end{array}$ & Participantes & $\begin{array}{l}\text { Logros } \\
\text { alcanzados }\end{array}$ & Punto critico & $\begin{array}{c}\text { Presupuesto / } \\
\text { Inversión }\end{array}$ & Responsable & $\begin{array}{c}\text { Medio de } \\
\text { verificación }\end{array}$ \\
\hline $\begin{array}{c}\text { Torneo } \\
\text { Nacional } \\
\text { de Ajedrez } \\
\text { clasificatorio } \\
\text { de Il división }\end{array}$ & $\begin{array}{c}\text { Sábados } \\
4,5 y \\
\text { Domingos } \\
11,12 \text { de } \\
\text { enero } \\
2020\end{array}$ & $\begin{array}{c}\text { Estudiantes } \\
\text { de: FAREM } \\
\text { CARAZO } \\
\text { SECUNDARIA } \\
\text { (Extensión) } \\
\text { Masc. 6, Fem. } \\
4\end{array}$ & $\begin{array}{c}\text { Clasificados: } \\
\text { Masc. } 3 \\
\text { Fem. } 3\end{array}$ & \begin{tabular}{|c|} 
Por falta de \\
transporte \\
no \\
participaron \\
5 de \\
nuestros \\
atletas.
\end{tabular} & $\begin{array}{l}\text { Cada Atleta } \\
\text { asumió sus } \\
\text { gastos }\end{array}$ & $\begin{array}{l}\text { FENICA } \\
\text { FENENIC }\end{array}$ & $\begin{array}{l}\text { Facebook, } \\
\text { FENANIC }\end{array}$ \\
\hline
\end{tabular}

Tabla 2 Estructura del informe trimestral de la disciplina de Ajedrez. Primer semestre 2020.

Fuente: informe de las actividades de ajedrez y tenis de mesa, correspondiente al primer semestre 2020. Elaborado por el maestro Sergio Iván Carranza, adecuado del formato del Plan Estratégico Institucional 2015-2019. Encabezan el informe en el formato original; la función sustantiva de la universidad, el objetivo estratégico, la estrategia y la acción estratégica.

universitaria, pero se requiere de una política de inversión o de gestión cultural y comunicativa en esta forma de extensión, que la potencialicen a nivel nacional e internacional y sobre todo, que logren mayor vinculación con las instituciones no académicas (instituciones, grupos sociales, asociaciones, etc.).

Se considera necesario, continuar analizando esta temática en las otras facultades de la universidad y desde la comunidad no universitaria, de tal modo que se logre responder las interrogantes: ¿Cómo es percibida la extensión cultural - artística y deportiva por parte de las comunidades receptoras (comunidad, instituciones estatales, empresa privada)? ¿Estarían dispuestas a establecer procesos de colaboración para el desarrollo de estas actividades y cómo? Dichas respuestas, podrían generar nuevas relaciones de diálogos y cooperación para el desarrollo de la extensión artística-cultural y deportiva en la UNANManagua y sus facultades regionales.

\section{Referencias bibliográficas}

- Asamblea Nacional de la República de Nicaragua. (1990). Ley de Autonomía de las Instituciones de Educación Superior. Gaceta $N^{\circ} 77$ del 05 de abril de 1990. Nicaragua.

- FAREM-CARAZO. (2015). Historia de la FAREM-Carazo. https://www.youtube.com/ watch?v=XJOV_gJ1674

- FAREM-CARAZO. (2018). Varios artículos. Boletín Torreón Universitario. Vol. 5 (1, 2, 3, 4 y 5).

- FAREM-CARAZO. (2019). Varios artículos. Boletín Torreón Universitario. Vol. 6 (5, 6, 8, 9, 10, 12, 13, $17,18,19,20$ y 21$)$.

- FAREM-CARAZO. (2020). Varios artículos. Boletín Torreón Universitario. Vol. 7 (2, 3, 5, 8, 13, 15 y 16).

- Fresan, M. (2004). La extensión universitaria y la educación pública. Reencuentro. $N^{\circ} 39$, Pp. 47-54. 
- Tünnermann, C. (2002). Los desafíos de la educación superior en Nicaragua. ENCUENTRO. 24 (63), Pp. 8-23.

- Universidad Nacional Autónoma de Nicaragua. (2018). Política de extensión y proyección universitaria 2017-2019 con su reforma. Estatutos aprobados por el consejo universitario en sesión 20-2018 del 31 de octubre de 2018.

\section{Entrevistas citadas}

- Cabezas, L. (07 de noviembre de 2020). Las actividades culturales y deportivas en la FAREMCarazo. (Guevara, W., entrevistador)

- Carranza, I. (07 de noviembre de 2020). Las actividades culturales y deportivas en la FAREMCarazo. (Guevara, W., entrevistador)

- Delgado, E. (07 de noviembre de 2020). Las actividades culturales y deportivas en la FAREMCarazo. (Guevara, W., entrevistador)

- Narváez, M. (07 de noviembre de 2020). Las actividades culturales y deportivas en la FAREMCarazo. (Guevara, W., entrevistador)

- Reyes, E. (07 de noviembre de 2020). Las actividades culturales y deportivas en la FAREMCarazo. (Guevara, W., entrevistador)

- Ríos, V. (07 de noviembre de 2020). Las actividades culturales y deportivas en la FAREM-Carazo. (Guevara, W., entrevistador)

- Rodríguez, F. (07 de noviembre de 2020). Las actividades culturales y deportivas en la FAREMCarazo. (Guevara, W., entrevistador)

- Urtecho, M. (07 de noviembre de 2020). Las actividades culturales y deportivas en la FAREMCarazo. (Guevara, W., entrevistador) 